elastography (SWE) is useful to distinguish pathological changes of the SG between early stage with normal salivary flow and advanced stage (EULAR2018). The present study we demonstrated that the tissue elasticity was increased due to inflammation and high viscosity in the SG at the early stage of SS with normal salivary flow comparing that in non-SS patients, but was decreased due to structural changes in the SG at the advanced stage of the disease. The SWE may be a useful tool for elucidation of early stage pathological changes of the SG when salivary gland functions are not impaired in SS.

Objectives: The aim of this study was to elucidate the usefulness of SWE in early-stage SS patients.

Methods: Seventeen non-SS patients and eighty patients who fulfilled the American College of Rheumatology (ACR)/European League Against Rheumatism (EULAR) classification criteria for SS were studied. SS patients were divided into three groups according to salivary flow using gum test (VL/SS: $<5 \mathrm{~mL} / 10 \mathrm{~min}$. ( $n=33)$, L/SS: $5-10 \mathrm{~mL} / 10 \mathrm{~min} . \quad(n=32)$ and N/SS: $\geqq 10 \mathrm{~mL} / 10 \mathrm{~min}$. $(n=15)$ ). All patients were examined SGUS by a single investigator who was blinded to device (TUS-A300; Canon Medical Systems, Tokyo, Japan) with a linear transducer $(7.5-10 \mathrm{MHz})$. The examination consisted of conventional B-mode US (US staging score), pulsed wave Doppler US (PD grading score) and SWE with quantitative assessment. US staging scores were assessed by glandular size, inhomogeneity and contrast of diagastric muscle (stage 0 to 3 ). PD grading scores were graded by pulsed wave pattern in pulsed wave Doppler US at the internal SG facial arteries (grade 0 to 2). With the region-of-interest (ROI) placed over the stiffest areas of the lesion on SWE, the quantitative mean of the elasticity values were measured by shear wave velocity (Vs: $\mathrm{m} / \mathrm{s}$ ) and elasticity (E: $\mathrm{kPa})$ for each lesion.

Results: The Vs and $\mathrm{E}$ values were correlated with US staging score $(r=-0.56, \quad p<0.001, \quad r=-0.58, \quad p<0.001)$ and $P D$ grading score $(r=-0.51$, $\mathrm{p}<0.001, \mathrm{r}=-0.52, \mathrm{p}<0.001)$ in all patients. There was no significant difference between non-SS and N/SS in early-stage SS by US staging score $(0.77 \pm 0.90$ vs $1.20 \pm 0.86)$ and $P D$ grading score $(0.18 \pm .053$ vs $0.53 \pm 0.19)$. However, the values of $V s$ and $E$ were highest in N/SS as compared with all groups, and significantly higher in N/SS than in non-SS (Vs: 1.73 \pm 0.18 vs $2.03 \pm 0.28 \mathrm{~m} / \mathrm{s}, \mathrm{p}<0.01, \mathrm{E}: 9.38 \pm 1.17$ vs $12.73 \pm 3.63 \mathrm{kPa}, \mathrm{p}<0.01$ ). Conclusion: The present study we demonstrated that the tissue elasticity was increased due to inflammation and high viscosity in the SG at the early stage of SS with normal salivary flow comparing that in non-SS patients. The SWE may be a useful tool for the differential diagnosis between patients with non-SS and early-stage SS with normal salivary flow in contrast to conventional SGUS.

Disclosure of Interests: None declared

DOI: 10.1136/annrheumdis-2019-eular.1518

\section{AB0521 EVALUATION OF LARYNGEAL IMPAIRMENT IN PATIENTS WITH SJÖGREN'S SYNDROME}

Benedikt Hofauer, Andreas Knopf, Carmen Unterhofer, Naglaa Mansour, Lara Kirschstein, Simone Graf. Technical University Munich, Otorhinolaryngology/ Head and Neck Surgery, Klinikum rechts der Isar, Munich, Germany

Background: Hyposalivation in patients with Sjögren's Syndrome is causative for oral dryness, one of the main symptoms of this disease. So far only little attention has been paid to a possible impairment of laryngeal functions due to the hyposalivation, even though, especially in younger and working patients, signs of laryngeal involvement often have an impact on the patient's quality of life.

Objectives: The objective of this study is to evaluate the laryngeal impairment in patients with Sjögren's Syndrome.

Methods: Patients with Sjögren's Syndrome diagnosed according to the AECG classification criteria were included in this study. Salivary function was evaluated by the measurement of the unstimulated salivary flow (USF). The laryngeal function was evaluated according to the European Laryngological Society protocol ${ }^{1}$, which includes five different items: perception (roughness, breathiness, hoarseness), videostroboscopy, acoustics (jitter, dysphonia severity index, DSI), aerodynamics and subjective rating by the patient (voice handicap index, $\mathrm{VHI}$ ).

Results: Eighteen patients (15 female, mean age 63 years) were included in the examination. Mean USF was $0.6 \mathrm{ml} / 5 \mathrm{~min}( \pm 0.5)$. The auditory-perceptual assessment resulted in slight and moderate deviance in 14 and 2 patients for roughness of the voice, in slight and moderate deviance in 12 and 3 patients for breathiness and in slight, moderate and severe deviance in 14, 3 and 1 patient for hoarseness. Evaluation of acoustics resulted in abnormal values in 12 patients (67\%). DSI revealed mild dysphonia in 7, moderate dysphonia in 6 and severe dysphonia in 1 patient. Evaluation of aerodynamics was abnormal in 12 (maximum phonation time) and 11 (phonation quotient) patients. Analysis of the VHI resulted in grade 0 in 5 patients (almost certainly not noticeable suffering), grade 1 in 8 patients (more likely unnoticeable than conspicuous suffering), grade 2 in 3 patients (more probably noticeable than not) and grad 3 in 2 patients (certainly noticeable suffering).

Conclusion: The evaluation of various laryngeal functions in patients with Sjögren's Syndrome unfolded an impairment of different aspects. Questions on laryngeal involvement should be implemented in the anamnesis of these patients and the application of screening methods should be further investigated.

\section{REFERENCES}

[1] Dejonckers $\mathrm{PH}$, et al. A basic protocol for functional assessment of voice pathology, especially for investigating the efficacy of (phonosurgical) treatment and evaluating new assessment techniques. Guideline elaborated by the Committee on Phoniatrics of the European Laryngological Society (ELS). Eur Arch Otorhinolaryngol 2001.

Disclosure of Interests: Benedikt Hofauer Consultant for: Consultant for Galvani Bioelectronics for the area of sleep disorders., Andreas Knopf: None declared, Carmen Unterhofer: None declared, Naglaa Mansour: None declared, Lara Kirschstein: None declared, Simone Graf: None declared

DOI: 10.1136/annrheumdis-2019-eular.5558

\section{AB0522 1 SEASONAL DIFFERENCE IN CEREBRAL STROKE FREQUENCY IN PATIENTS WITH SYSTEMIC LUPUS ERYTHEMATOSUS}

Wen-Nan Huang ${ }^{1}$, Yi-Ming Chen ${ }^{1,2}$, Yi-Hsing Chen ${ }^{1}$, Wei-Ting Hung ${ }^{1}$, YuWan Liao ${ }^{1} .{ }^{1}$ Taichung Veterans General Hospital, Division of Allergy, Immunology and Rheumatology, Taichung, Taiwan, Republic of China; ${ }^{2}$ Taichung Veterans General Hospital, Department of Medical Research, Taichung, Taiwan, Republic of China

Background: The incidence of stroke is well-known as increased in winter season in general population, but seasonal difference in the systemic lupus erythematosus remain unclearified.

Objectives: The aim of study was to assess seasonal difference in cerebral stroke among patients with systemic lupus erythematosus.

Methods: We conducted a retrospective case-control study analyzing hospital database of a tertiary referral center in Taiwan. From January, 2002 to December 2017, subjects with systemic lupus erythematosus and cerebral stroke were enrolled. To examine the seasonal incidence of cerebral stroke, we analyzed data from the date of CT scan/MRI/MRA. The onset time was divided into four seasons (according Local season classification): spring (March, April, May), summer (June, July, August), autumn (September, October, November), and winter (December, January, February).

Results: In total, 4313 systemic lupus erythematosus patients were identified, 261 patients with cerebral stroke. Age of patients with cerebral stroke ranged 27 between 77 years, a mean age was 55-62 years. Female was predominant. Disease duration was longer in ischemia stroke group (4.8 years). According CT or MRI results, divided 4 types (Ischemia stroke (IS), ICH, SAH, SDH), 83\% (150/180) cerebral stroke was ischemia stroke. $8.3 \% \mathrm{ICH}, 5.5 \% \mathrm{SAH}, 2.8 \% \mathrm{SDH}$ found respectively. We noted a higher incidence of SLE with ischemia stoke in summer season $(38.7 \%)$, the lowest incidence of ischemia stroke in winter season $(18.7 \%)$. Patient with $\mathrm{ICH}$ has similar trend. But not found in $\mathrm{SAH}$ and $\mathrm{SDH}$

Conclusion: Incidence of systemic lupus erythematous with cerebral stroke varied in different pattern. The present findings suggested an increase in the incidence of Ischemia stroke and $\mathrm{ICH}$ in summer season. Awareness of risk factors (esp. antiphsopholipid antibodies) was essential to prevent cerebral stroke in SLE.

Table Seasonal difference in different types of cerebral stroke

\begin{tabular}{lcccc}
\hline & $\mathrm{IS}(\mathrm{n}=150)$ & $\mathrm{ICH}(\mathrm{n}=15)$ & $\mathrm{SAH}(\mathrm{n}=10)$ & $\mathrm{SHA}(\mathrm{n}=5)$ \\
\hline Age & 60 & 59.0 & 60.5 & 55.0 \\
& $(46.8-75.0)$ & $(35.0-68.0)$ & $(43.3-77.3)$ & $(27.0-71.0)$ \\
Gender\% (F/M) & $73.3 / 26.7$ & $66.7 / 33.3$ & $90.0 / 10.0$ & $60.0 / 40.0$ \\
Disease duration & $4.8(0.3-8.7)$ & $2.5(0.0-5.5)$ & $3.9(0.0-11.6)$ & $0.0(0.0-11.5)$ \\
Season & & & & \\
Spring (3-5) & $21.3 \%$ & $26.7 \%$ & $20.0 \%$ & $60.0 \%$ \\
Summer (6-8) & $38.7 \%$ & $53.3 \%$ & $20.0 \%$ & $20 \%$ \\
Autumn (9-11) & $21.3 \%$ & $6.7 \%$ & $30.0 \%$ & $20 \%$ \\
Winter (12-2) & $18.7 \%$ & $13.3 \%$ & $30.0 \%$ & $0 \%$ \\
\hline
\end{tabular}


Disclosure of Interests: None declared

DOI: 10.1136/annrheumdis-2019-eular.7489

\section{AB0523 MENOPAUSE IN AN EGYPTIAN COHORT OF SYSTEMIC LUPUS ERYTHEMATOSUS PATIENTS: EFFECT OF THE DISEASE}

Samah A. Elbakry, Safaa A. Hussein, Rasha M. Hamouda, Marina W. Naguib. Internal Medicine Department, Division of Rheumatology, Ain Shams University, Cairo, Egypt

Background: Systemic lupus erythematosus (SLE) is a chronic systemic autoimmune disease that mainly affects females in the reproductive age ${ }^{1}$. Although SLE generally emerges during reproductive ages, it was found that lupus patients experience menopause at younger age than the general population; however, whether the occurrence of menopause at a younger age in lupus patients results from the gonadotoxic effects of Cyclophosphamide treatment or from an autoimmune-mediated ovarian injury is debated ${ }^{2}$

Objectives: To identify menopause characteristics in an Egyptian cohort of women with SLE with effect of the disease on menopausal symptoms and the characteristics of disease activity and disease damage in perimenopausal and post-menopausal patients.

Methods: In this cross-sectional observational study, data of 120 consecutive SLE female patients who fulfilled the 2012 ACR/SLICC criteria $^{3}$, above the age of 35 . Disease activity was assessed by using the SLE disease activity index (SLEDAI) ${ }^{4}$, and accumulated damage was assessed by Systemic Lupus International Collaborative Clinics/American College of Rheumatology Damage Index (SLICC/ACR DI) ${ }^{5}$. Laboratory assessment was done to all patients including follicle stimulating hormone (FSH) and luteinizing hormone (LH) blood levels.

Results: The mean age of the patients was $45.067 \pm 8.211$ years and median disease duration was $5.074 \pm 5.567$ years $(\min 0.08-\max 21$ years). It was found that the mean of total SLEDAI score was $4.667 \pm$ 3.537 and mean of total SLICC/ACR damage index was $0.633 \pm 0.819$. Of 120 patients $20 \%$ had premature menopause, $29.17 \%$ had natural menopause and $50.83 \%$ were still menstruating (table 1). It was found that the age at menopause (either natural or premature menopause) ranged from 26 to 54 years with the mean $45.170 \pm 7.278$ years. There was a statistically significant negative correlation between $\mathrm{LH}$ and total SLEDAI $(r=-0.178, P=0.052)$. It was also found a statistically significant positive correlation between FSH and cumulative Cyclophosphamide dose $(r=0.440, P<0.001)$ and between $\mathrm{LH}$ and cumulative Cyclophosphamide dose $(r=0.214, P=0.046)$ (table 2$)$.

Table 1. Menstrual status:

\begin{tabular}{lcc}
\hline & \multicolumn{2}{c}{ Positive } \\
\cline { 2 - 3 } & $\mathrm{N}$ & $\%$ \\
\hline Premature & 24 & 20.00 \\
menopause & & \\
Natural menopause & 35 & 29.17 \\
Menstruating & 61 & 50.83 \\
\hline
\end{tabular}

Conclusion: SLE has a reproductive and hormonal impact on female patients, either because of disease activity or due to pharmacological adverse effects. The mean age at menopause (either natural or premature menopause) is 45 years. High $\mathrm{LH}$ is associated with lower disease activity. High cumulative Cyclophosphamide dose is associated with high $\mathrm{FSH}$ and $\mathrm{LH}$.

\section{REFERENCES}

[1] Mills JA. Systemic lupus erythematosus. New Engl J Med 1994; 330: 1871-1879.

[2] Sammaritano LR. Menopause in patients with autoimmune diseases. Autoimmun Rev. 2012; 11(6-7).

[3] Petri M., Orbai AM., Alarcón GS, et al. Derivation and Validation of the Systemic Lupus International Collaborating Clinics Classification Criteria for Systemic Lupus Erythematosus. Arthritis Rheum. 2012; 64: 2677-86.

[4] Bombardier C, Gladman D, Urowitz M, et al. Derivation of the SLEDAI. A disease activity index for lupus patients. The Committee on Prognosis Studies in SLE. Arthritis Rheum 1992, 35, 630-640.

[5] Gladman D, Ginzler E, GoldsmithC, et al. The development and initial validation of the Systemic Lupus International Collaborating Clinics/American College of Rheumatology damage index for systemic lupus erythematosus. Arthritis Rheum. 1996; 39: 363-369.
Table 2. Correlation study between FSH \& LH and Cyclophosphamide:

\begin{tabular}{|c|c|c|c|c|}
\hline \multicolumn{5}{|l|}{ Correlations } \\
\hline & \multicolumn{2}{|c|}{$\mathrm{FSH}(\mathrm{mlU} / \mathrm{ml})$} & \multicolumn{2}{|c|}{$\mathrm{LH}(\mathrm{mlU} / \mathrm{ml})$} \\
\hline$\overline{\mathrm{R}}$ & $\begin{array}{c}\mathrm{P}- \\
\text { value }\end{array}$ & $\bar{R}$ & $\begin{array}{c}P_{-} \\
\text {value }\end{array}$ & \\
\hline Cumulative & 0.440 & $<$ & 0.214 & $0.046^{*}$ \\
\hline $\begin{array}{l}\text { Cyclophosphamide } \\
\text { (mg) }\end{array}$ & & $0.001^{*}$ & & \\
\hline
\end{tabular}

Disclosure of Interests: None declared

DOI: 10.1136/annrheumdis-2019-eular.1226

\section{AB0524 $\quad$ SERUM BAFF AND APRIL AS CANDIDATE BIOMARKERS IN SYSTEMIC LUPUS ERYTHEMATOSUS (SLE): A PROSPECTIVE FOLLOW-UP STUDY}

Selma Sarı ${ }^{1}$, Suzan Çınar ${ }^{2}$, Bahar Artim-Esen ${ }^{3}$, Ahmet Gul $^{3}$, Mahdume Lale Ocal ${ }^{3}$, Gunnur Deniz ${ }^{2}$, Murat Inanc ${ }^{3} .{ }^{1}$ Istanbul University, Istanbul Faculty of Medicine, Internal Medicine, Istanbul, Turkey; ${ }^{2}$ Istanbul University, Aziz Sancar Institute of Experimental Medicine, Immunology, Istanbul, Turkey, ${ }^{3}$ Istanbul University, Istanbul Faculty of Medicine, Rheumatology, Istanbul, Turkey

Background: BAFF and APRIL are cytokines involved in B cell development and they take place in the pathogenesis of SLE.

Objectives: The aim of this study was to investigate the relationship between serum BAFF/APRIL levels with clinical features and disease activity in SLE patients.

Methods: We included 79 patients with SLE (ACR criteria) and 27 healthy controls into the study. Serum BAFF and APRIL levels were assessed by ELISA. In 19 patients with active disease at the time of the assessment BAFF/APRIL levels were reassessed at least 6 months later (mean 7.8 months) and disease activity was evaluated by SLEDAI. New renal involvement was observed in 16 patients during the study and renal involvement was previously detected in 12 patients.

Results: Although both BAFF (median 0.7 vs $0.41 \mathrm{ng} / \mathrm{ml}$ ) and APRIL (median 2.3 vs $1.05 \mathrm{ng} / \mathrm{ml}$ )levels were higher in patients with SLE compared to the control group ( $p<0.001$ ), no correlation was found between BAFF/APRIL levels and SLEDAI scores. Only 9 patients (11.4\%) had both BAFF and APRIL in normal range (95\% confidence interval). When patients were grouped according to disease activity as no activity (SLEDAl $=0$ ), low disease activity and active disease, there was no difference in BAFF/APRIL levels between groups. Serum BAFF levels were higher in patients with renal disease activity (median $0.94 \mathrm{ng} / \mathrm{ml}$ vs 0.61 $\mathrm{ng} / \mathrm{ml}, \mathrm{p}=0,01)$, and there was a positive correlation between APRIL levels and proteinuria $(r=0.42, p=0,02)$. There was no association between BAFF/APRIL levels and anti-dsDNA positivity but a weak inverse correlation was observed between BAFF and C3 levels $(r=0.25, P=0.02)$. No correlation was found between BAFF/APRIL levels and renal SLEDAI score, renal histopathology activity and chronicity index scores. In the active disease group after follow-up, there was no significant change in BAFF (from 1,63 ng/ml to $1,2 \mathrm{ng} / \mathrm{ml}$ ) and APRIL levels (from 2,11 ng/ml to $2,31 \mathrm{ng} / \mathrm{ml}$ ).

Conclusion: BAFF/APRIL levels were found to be significantly higher in patients with SLE compared to controls, but no association with disease activity was found. BAFF levels are correlated with decreased C3 levels. These results suggest that both cytokines are involved in the pathogenesis of SLE, and that serum BAFF and APRIL levels can be valuable as a biomarker in SLE especially in patients with renal activity. Long-term studies on the effect of treatment are needed.

Disclosure of Interests: None declared

DOI: 10.1136/annrheumdis-2019-eular.4671

\section{AB0525 ANTI-SMITH ANTIBODY IS ASSOCIATED WITH DISEASE ACTIVITY IN PATIENTS WITH NEW-ONSET SYSTEMIC LUPUS ERYTHEMATOSUS}

Seungmin Jung, Juyoung Yoo, Sungsoo Ahn, Sangwon Lee, Jason Jungsik Song, Yongbeom Park. Yonsei University College of Medicine, Internal Medicine, Seoul, Korea, Rep. of (South Korea)

Background: Anti-Smith (Sm) antibody is a highly specific antibody for systemic lupus erythematosus (SLE). Despite the remarkable specificity of anti-Sm antibodies for SLE, the association between anti-Sm antibody level and the clinical manifestation of SLE is still unclear.

Objectives: We aimed to evaluate the association between anti-Sm antibodies and disease activity in patients with new-onset SLE. 